Chirurg 2018 $89: 928$

https://doi.org/10.1007/s00104-018-0729-8

Online publiziert: 14 . September 2018

(c) Springer Medizin Verlag $\mathrm{GmbH}$, ein Teil von Springer Nature 2018

\section{CrossMark}

M. Schrempf · M. Anthuber

Klinik für Allgemein-, Viszeral- und Transplantationschirurgie, Klinikum Augsburg, Augsburg, Deutschland

\title{
Ist eine leitliniengerechte Händedesinfektion auf Intensivstationen möglich?
}

Händedesinfektion und die Abweichung von der empfohlenen Dauer erfasst.

Stahmeyer JT, Lutze B, von Lengerke T et al (2017) Hand hygiene in intensive care units: a matter of time? J Hosp Infect 95:338-343. https://doi.org/10.1016/j.jhin.2017.01.011

Hintergrund. Nosokomiale Infektionen und die Entwicklung multiresistenter Keime sind zu einer großen Herausforderung in der modernen Medizin geworden. Sie sind für ca. 20.000 bis 30.000 Todesfälle in Deutschland verantwortlich und verursachen mit geschätzten 13-24 Mrd. € allein in Europa immense Kosten im Gesundheitssystem. Eine der einfachsten, wichtigsten und effektivsten Hygienemaßnahmen stellt die Händehygiene dar. Die Compliance für diese simple Maßnahme ist jedoch in vielen Studien auf ärztlicher wie pflegerischer Seite erschreckend gering. Die vorgestellte Arbeit gibt Hinweise darauf, dass eine leitliniengerechte Händehygiene vor allem durch Zeit- und Personalmangel erheblich erschwert wird.

Methoden. Diese Beobachtungsstudie wurde auf einer internistischen (ICU) und einer chirurgischen (SICU) Intensivstation in Deutschland durchgeführt. Die Anzahl der beobachteten WHOIndikationen zur Händedesinfektion ("hand hygiene opportunities HHOs“; vor Patientenkontakt - vor einer aseptischen Tätigkeit - nach Kontakt mit potenziell infektiösem Material - nach Patientenkontakt - nach Kontakt mit der unmittelbaren Patientenumgebung) wurde mit der Anzahl der tatsächlich durchgeführten Händedesinfektionen verglichen und zusätzlich die Dauer der
Ergebnisse. Die Gesamtbeobachtungszeit betrug $144 \mathrm{~h}$. In dieser Zeit wurden insgesamt 1896 HHOs registriert. Durchschnittlich wurden zwischen 7:00 und 19:00 Uhr 158 HHOs pro Patient gemessen (134 ICU, 182 SICU). Die extrapolierte Anzahl an HHOs pro Tag und Patient betrug 218 (ICU) bzw. 271 (SICU). Die Compliance betrug 42,6\% (Pflegepersonal 42,1\%, ärztliches Personal 42,5\%, sonstiges Personal 55,9\%). Die durchschnittliche Dauer der ausgewerteten Händedesinfektionen lag mit 7,6 s deutlich unter den empfohlenen $30 \mathrm{~s}$. 94,3\% der Händedesinfektionen dauerten weniger als $15 \mathrm{~s}$. Von 1896 beobachteten HHOs erfolgte in nur 2 Fällen eine korrekte Händedesinfektion. Die theoretisch erforderliche Zeit für Händehygiene pro Patient und Tag betrug 95 min (ICU) bzw. 104 min (SICU).

Diskussion und Fazit. In dieser Arbeit erfolgte bei gerade einmal 2 von 1896 HHOs eine korrekte Händehygiene. Dieses katastrophale Ergebnis überrascht nur wenig und dürfte auf Normalstationen ähnlich ausfallen. Es wird deutlich, dass korrekt durchgeführte Hygienemaßnahmen einen erheblichen Zeit- und damit auch Personalaufwand verursachen. Der Hauptanteil $(81,1 \%)$ der HHOs entfällt dabei auf das Pflegepersonal. Legt man den durchschnittlichen Pflegeschlüssel von 2,47 Patienten pro Pflegekraft auf Intensivstationen von Universitätsklinika zugrunde, so bedeutet dies, dass eine Pflegekraft während einer 8-stündigen Schicht $78 \mathrm{~min}$ (ICU) bzw. $86 \mathrm{~min}$
(SICU) nur für die Händedesinfektion aufbringen müsste. Dies entspricht 16-18\% der Arbeitszeit und steht in starkem Kontrast zur tatsächlich für die Händehygiene aufgebrachten Zeit von 9,1-10 min (1,9-2,1\% der Arbeitszeit). Es wird nicht klar, ob Politik und Klinikträger sich dieser Situation bewusst sind. Jedenfalls scheint der zusätzliche Zeit- und Personalaufwand für eine korrekt durchgeführte Händedesinfektion keinen angemessenen Niederschlag in den Personalschlüsseln zu finden. Der Wert der berechtigten Diskussion um Screening- und Isolationsmaßnahmen sowie den übermäßigen und falschen Einsatz von Antibiotika wird in höchstem Maße infrage gestellt, wenn selbst einfachste, aber doch hoch effektive Hygienemaßnahmen aufgrund von Zeitund Personalmangel nicht durchgeführt werden bzw. nicht durchgeführt werden können.

\section{Korrespondenzadresse}

\section{Prof. Dr. M. Anthuber}

Klinik für Allgemein-, Viszeral- und

Transplantationschirurgie, Klinikum Augsburg Stenglinstr. 2, 86156 Augsburg, Deutschland matthias.anthuber@klinikum-augsburg.de

Interessenkonflikt. M. Schrempf und M. Anthuber geben an, dass kein Interessenkonflikt besteht. 